\title{
PREVALÊNCIA E FATORES ASSOCIADOS À BACTEREMIA NOS PORTADORES DE COLECISTITE AGUDA LITIÁSICA
}

\author{
André luciano Baitello*, Ramiro Colleoni Neto, Benedito Herani Filho, José Antônio Cordeiro, \\ Antônia M. O. Machado, Moacir F. Godoy, felipe franco Pinheiro Gaia \\ Trabalho realizado na disciplina de Gastroenterologia Cirúrgica, Universidade Federal de São Paulo, \\ Escola Paulista de Medicina, São Paulo, SP e departamento de Cirurgia Geral, Fundação Faculdade \\ de Medicina de São José do Rio Preto, São Paulo, SP
}

RESUMO - OBJETIVO. Determinar a prevalência de bacteremia e os fatores associados no período pré-operatório em portadores de colecistite aguda litiásica e analisar comparativamente as complicações e mortalidade nos pacientes com e sem bacteremia, que foram submetidos a colecistectomia de urgência.

Métodos. Foram estudados, prospectivamente, $5 \mathbf{I}$ pacientes com diagnóstico histopatológico de colecistite aguda litiásica. 0 sistema Bactec foi o método utilizado para a detecção de bacteremia. As médias dos grupos foram analisadas quanto às variáveis clínicas e laboratoriais, relacionando-as com a bacteremia.

Resultados. A prevalência de bacteremia no pré-operatório foi de $15,68 \%$, e a idade $(p=0,024)$, a freqüência cardíaca $(p=0,026)$, a frequêencia respiratória $(p=0,028)$, a creatinina $(p=0,028)$ e a presença da SIRS (Síndrome da Resposta Inflamatória Sistêmica, $p=0,016)$ associaram-se positivamente com a bacteremia. Nos portadores de bacteremia verificou-se um óbito, maior número de complicações gerais $(p=0,045)$ e infecciosas $(p=0,039)$ e maior tempo de internação $(p<0,005)$.

Conclusāo. Nessa amostra, utilizando-se o sistema Bactec, a prevalência de bacteremia foi considerável. Fatores clínicos e laboratoriais estão associados à bacteremia e a sua presença associa-se à maior gravidade dos pacientes e pior prognóstico em relação às complicaçóes no pós-operatório nos portadores de colecistite aguda litiásica.

Unitermos: Bacteremia. Prevalência. Colecistite aguda. SIRS e complicações.

\section{INTRODUÇÃO}

A patogênese da colecistite aguda calculosa inicia-se na grande maioria das vezes pela obstrução do ducto cístico ou infundíbulo vesicular por um cálculo, desencadeando uma resposta inflamatória pela combinação de três fatores: mecânico, químico e infeccioso. 0 fator mecânico produz elevação da pressão intraluminal e distensão do órgão, que culmina na isquemia da vesícula biliar. A inflamação química ocorre pela liberação de vários mediadores inflamatórios, entre eles lisolecitina, fosfolipase A e prostaglandinas, resultando na lesão direta da mucosa! .

A contribuiç̧ão dos agentes bacterianos nesse processo inflamatório é variável, pois de 50\% a $70 \%$ dos pacientes com colecistite aguda litiásica apresentam crescimento de bactérias no conteúdo biliar (bactibilia) 2,3,4. As bactérias

*Correspondência: Condomínio Green Park - Jd. Vivendas Rua José Felipe Antonio, 303 - Apto. 43 - Bloco 7 CEP: 15.090-430 - São José do Rio Preto - SP Telefone: (17) 210-1437 predominantemente envolvidas são enterobactérias (Escherichiacoli, Klebsiella sp, Enterococcus, bacterióidese Clostridium sp) $)^{5,6}$. A presença de bactibilia determina um foco primário de infecção e pode propiciar condições para o desenvolvimento de bacteremia nos portadores de colecistite aguda litiásica?.

O diagnóstico de bacteremia consiste na detecção de bactérias viáveis na corrente sangüínea ${ }^{8}$. $O$ modo mais sensível de detecção da mesma é pela realização de hemoculturas 9,10 . A presença da bacteremia pode estar associada a complicações supurativas a distância?, acentua a resposta inflamatória sistêmica $^{10}$, e determina maior morbidade e mortalidade ${ }^{11,12}$. Estudos com pacientes que necessitam de tratamento clínico ou cirúrgico demonstram a importância da bacteremia no prognóstico dos pacientes estudados, levando a um aumento da mortalidade e complicações ${ }^{11,13}$.

A colecistite aguda e a bacteremia podem, a partir da liberação de vários mediadores humorais, desencadear a Síndrome da Resposta Inflamatória Sistêmica (SIRS), cujos critérios diagnósticos atualmente estão bem estabelecidos ${ }^{10}$ e são de fácil utilização, podendo ser utilizados à beira do leito.

Métodos capazes de receber maiores volumes de sangue mostram-se mais sensíveis na detecção de bacteremia ${ }^{14,15,16}$. Um desses é o sistema Bactec 9240® (Becton Dickinson and Company), método automatizado utilizado para pesquisa de bactérias no sangue e na bile. A utilização desse método no diagnóstico de bacteremia já está difundida e seu uso consagrado ${ }^{17}, 18$.

Apesar da bacteremia na colecistite aguda ser provavelmente um evento intermitente ${ }^{18}$, sua prevalência e significado clínico em pacientes com colecistite aguda litiásica não estão definitivamente determinados.

Utilizando-nos de um estudo prospectivo em pacientes portadores de colecistite aguda litiásica, tendo o exame histopatológico como teste padrão definitivo para o diagnóstico e usando técnica moderna e sensível de detecção de bacteremia, decidimos determinar a prevalência e os fatores associados a bacteremia nesses pacientes. 
BATtello AL et AL.

\section{Métodos}

Foram estudados, prospectivamente, 51 pacientes admitidos pelo Serviço de Cirurgia Geral e Emergência do Hospital de Base/Faculdade Estadual de Medicina de São José do Rio Preto, em um período de 36 meses compreendidos entre novembro de 1999 e novembro de 2002. Foram incluídos neste protocolo doentes portadores de colecistite aguda litiásica, submetidos a colecistectomia aberta até 72 horas após admissão no serviço. Foram adotados como critérios de exclusão os pacientes portadores de pancreatite aguda biliar; coledocolitíase e/ou colangite associadas a colecistite aguda litiásica com diagnóstico estabelecido no período pré-operatório; o uso de antibióticos até sete dias prévios que antecederam admissão hospitalar; os portadores de colecistite alitiásica, em uso de drogas imunossupressoras ou portadores da Síndrome de Imunodeficiência Adquirida (AIDS); pacientes com infecções outras que não a da vesícula biliar nas últimas quatro semanas; tratamento dialítico concomitante e história prévia de transplantes de órgãos.

O diagnóstico de colecistite aguda litiásica foi baseado nos dados obtidos da história clínica, do exame físico, de exames laboratoriais e ultrasonografia abdominal. $\bigcirc$ exame considerado padrão de referência para este diagnóstico foi o histopatológico, que mostrou a presença de infiltrado polimorfonuclear e/ou achados de necrose, edema e hemorragia de uma das camadas da parede da vesícula biliar19,20.

Os dados da avaliação clínica e laboratorial associados com a presença de bacteremia foram os dados de identificação do paciente, os utilizados rotineiramente no diagnóstico de colecistite aguda litiásica e SIRS, sendo os seguintes: da identificação: idade, sexo; da história clínica: anorexia, febre, calafrios, náuseas, vômitos, história prévia de dor biliar; do exame físico: freqüência cardíaca, freqüência respiratória, pressão arterial média, temperatura, sinal de Murphy, vesícula palpável, tumor palpável no hipocôndrio direito, dor à descompressão súbita dolorosa; e dos exames laboratoriais: dosagem de creatinina, glicemia, bilirrubinas, aspartatoamino-transferase (AST), aspartatoalanino-transferase (ALT), fosfatase alcalina, gamaglutamil-transferase, amilase, hematócrito, hemoglobina, contagem de leucócitos e proteína C reativa; e o preenchimento dos critérios de SIRS.
As amostras de sangue foram obtidas por punção de veia periférica do antebraço, após antissepsia da pele do membro superior com álcool etílico a $70 \%$ e iodo povidine a 10\%. Essas amostras foram realizadas logo após o diagnóstico de colecistite aguda ter sido estabelecido no período pré-operatório através do exame clínico e ultra-som do abdome. Foram colhidas duas amostras de sangue, com intervalo de 60 minutos entre as punções. Em cada amostra foram coletados $20 \mathrm{ml}$ de sangue e distribuídos em dois frascos para hemocultura, sendo um para bactérias aeróbias e outro para bactérias anaeróbias. Após a coleta, os frascos foram imediatamente encaminhados ao laboratório e inseridos no sistema Bactec $9240 \AA$.

Os frascos utilizados como meio para as hemoculturas e cultura do conteúdo biliar foram os do sistema Bactec $9240 \AA$ (Becton Dickinson and Company), com resina para adsorção de antibióticos, o Bactec Plus Aerobic $\AA^{\circledR}$ e o Bactec Plus ${ }^{\circledR}$ Anaerobic $($ ). Os frascos Bactec Plus - Aerobic/F (Becton Dickinson) contêm um meio de cultura composto de caldo de soja tripsicaseína, hemina, polianetol, sulfato de sódio e resinas aniônicas e catiônicas com ação neutralizante de antibióticos. Todos os frascos continham vácuo e dióxido de carbono. Os frascos para anaeróbios, Bactec Plus - Anaerobic/F, são suplementados com vitaminas $K$, extrato de leveduras e redutores, tendo atmosfera suplementada com nitrogênio.

Os frascos de cultura foram imediatamente encaminhados para o Laboratório Central do Hospital de Base da Faculdade Estadual de Medicina de São José do Rio Preto, e incubados no sistema Bactec $9240 ®$ (Becton Dickinson and Company), em temperatura constante, onde permaneceram por cinco dias no máximo. Todos os frascos da estante do sistema são avaliados periodicamente a cada dez minutos. Culturas com crescimento de microrganismos são imediatamente sinalizadas por um indicador luminoso na frente do aparelho e mostradas no monitor do computador acoplado ao sistema, e também por um alarme sonoro.

Se a leitura do sistema Bactec $9240 \AA$ (Becton Dickinson and Company) apresentou - crescimento de bactérias, foi realizado bacterioscopia pelo método de Gram, e o subcultivo dos frascos das hemoculturas foi semeado em duas placas de Petri, uma com ágar-sangue e outra com ágar-chocolate enriquecido, que foram levadas a uma estufa de $\mathrm{CO}^{2}$ a $36,5^{\circ} \mathrm{C}$ por 24 horas. Se não ocorreu isolamento de bactérias, as placas foram novamente incubadas por mais 24 horas. Se após 72 horas não ocorreu a formação de colônias, o resultado foi considerado negativo. Havendo formação de colônias, à identificação da bactéria, segue a rotina do Laboratório Central da Faculdade Estadual de Medicina de São José do Rio Preto, e em paralelo com o sistema autoSCAN®/MICROSCAN®) (Baxter Instruments). $O$ autoSCAN $B / M I C R O S C A N \otimes$ é sistema automatizado que utiliza método colorimétrico para identificar as bactérias e realizar também o teste de sensibilidade das mesmas aos antimicrobianos.

Foram consideradas no diagnóstico de bacteremia bactérias identificadas em uma ou mais amostras de hemoculturas. Foram excluídas as consideradas contaminantes.

Foram considerados contaminantes e excluídos do grupo de pacientes bacterêmicos aqueles em que se encontrava, em uma única hemocultura, o crescimento de bactérias tipicamente da flora cutânea, como Staphylococcus epidermidis, Streptococcus viridanse Corynebacterium spp $p^{21,22, \text { e }}$ as culturas de bile não evidenciavam o isolamento dessas bactérias.

No período pós-operatório hospitalar, os pacientes foram examinados diariamente, atentando-se para complicações infecciosas do sítio cirúrgico e fora dele (pneumonia e infecção do trato urinário) e complicações não infecciosas (atelectasia, seroma, infarto agudo do miocárdio, insuficiência renal aguda, hemorragia digestiva e outras). Após a alta hospitalar, os pacientes foram avaliados rotineiramente no 10\% $30 \% / 90^{\circ}$ dias de pós-operatório.

Os testes estatísticos foram realizados pelo programa Minitab for Windows, versão 12.23. Para análise dos pacientes bacterêmicos e não bacterêmicos quanto às variáveis do tipo contínua, e com distribuição normal, foi utilizado o teste T. Para análise das variáveis contínuas com distribuição não gaussiana foi utilizado o teste de Mann-Whitney. Para comparação de duas proporções foi utilizado o teste exato de Fisher. Uma diferença foi considerada significante quando valor $p \leq 0,05$. 


\section{Resultados}

A bacteremia foi detectada em 8 de 51 pacientes, evidenciando uma prevalência de 15,68\% nos portadores de colecistite aguda litiásica.

Procuramos determinar as repercussões clínicas a partir da análise, comparativa entre os dados demográficos, clínicos e laboratoriais. Assim, para fins de análise os pacientes foram divididos em dois grupos, quanto à presença ou não de bacteremia.

Não foram observadas diferenças significantes entre os grupos com e sem bacteremia em relação ao sexo $(p=0,13)$; dados de história clínica: febre e calafrios $(p=0,13)$, anorexia ( $p$ $=0,58)$, náuseas e vômitos $(p=0,17)$, história de dor biliar semelhante prévia $(p=1,00)$.

Há evidência de diferença significante na média de idades nos grupos com bacteremia e sem bacteremia $(p=0,024)$. Estes dados podem ser observados na Tabela 1.

Dos sinais de exame físico, observaram-se diferenças significantes nos dados de freqüência cardíaca $(p=0,026)$ e freqüência respiratória ( $p=0,028)$, conforme Tabela 2 .

0 resultado observado quanto ao diagnóstico de SIRS mostra-se significante quanto à presença de bacteremia, conforme Tabela 3.

Dos dados do exame do abdome não se observaram diferenças significantes nos sinais analisados: sinal de Murphy ( $p=0,57$, vesícula palpável $(p=0,51)$, "tumor" palpável $(p=1,00)$, dor à descompressão brusca positiva $(p=1,00)$ (Tabela 4).

Houve diferença significante em complicações infecciosas entre os grupos analisados, como mostrado na Tabela 5.

Seis pacientes apresentaram complicações no grupo bacteremia, enquanto 14 pacientes do grupo sem bacteremia apresentaram complicações, evidenciando um valor $p=0,045$ (Teste exato de Fisher).

Observou-se no grupo bacteremia um tempo médio de internação de nove dias, variando de 4 a 36 dias. No grupo sem bacteremia, o tempo médio de internação foi de quatro dias, variando de 3 a 15 dias, assim os resultados mostram uma diferença altamente significante com $p<0,005$.

Um paciente do grupo bacteremia faleceu, devido a complicações cardíacas, bloqueio atrioventricular total, infarto agudo do miocárdio e choque cardiogênico, seguido de falência de múltiplos órgãos.
Tabela I - Distribuição segundo idade nos grupos com e sem bacteremia

\begin{tabular}{|c|c|c|c|c|}
\hline \multirow[b]{2}{*}{ Faixa etária (anos) } & \multicolumn{2}{|c|}{ Bacteremia } & \multicolumn{2}{|c|}{ Sem bacteremia } \\
\hline & $\mathbf{N}$ & $\%$ & $\mathbf{N}$ & $\%$ \\
\hline $20 \mid-30$ & 0 & 0,00 & 8 & 18,60 \\
\hline $30 \mid-40$ & | & 12,50 & 6 & 13,95 \\
\hline $40 \mid-50$ & 0 & 0,00 & 6 & 13,95 \\
\hline $50 \mid-60$ & 0 & 0,00 & 9 & 20,93 \\
\hline $60 \mid-70$ & 3 & 37,50 & 8 & 18,60 \\
\hline $70 \mid-80$ & 2 & 25,00 & 6 & 13,95 \\
\hline $80 \mid-$ & 2 & 25,00 & 0 & 0,00 \\
\hline Total & 8 & 100,00 & 43 & 100,00 \\
\hline Média de idades- Desvio padrão & & & & \\
\hline
\end{tabular}

Teste t- (valor $p=0,024$ )

Tabela 2 - Distribuição das medianas dos sinais vitais nos grupos com e sem bacteremia

\begin{tabular}{|c|c|c|c|}
\hline \multirow[b]{2}{*}{ Sinais Vitais } & \multicolumn{2}{|c|}{ Mediana dos grupos } & \multirow[b]{2}{*}{ p(Mann Whitney) } \\
\hline & Bacteremia & Sem bacteremia & \\
\hline Freqüência cardíaca(bpm) & 108,0 & 990,0 & 0,026 \\
\hline Freqüência respiratória(mpm) & 24,8 & 20,0 & 0,028 \\
\hline Pressão arterial média (mmhg) & 96,0 & 96,0 & 0,28 \\
\hline Temperatura $\left({ }^{\circ} \mathrm{C}\right)$ & 37,8 & 37,8 & 0,30 \\
\hline
\end{tabular}

Tabela 3 - Distribuição da prevalência de SIRS nos grupos com e sem bacteremia

\begin{tabular}{|c|c|c|c|c|}
\hline \multirow{3}{*}{ SIRS } & & Bacteremia & Sem bacteremia & $\mathrm{p}$ \\
\hline & Presente & 7 & 16 & 0,016 \\
\hline & Ausente & | & 27 & \\
\hline
\end{tabular}

Teste exato de Fisher (valor $p=0,016$ )

Tabela 4 - Distribuição dos resultados dos exames laboratoriais, média e desvio padrão nos grupos com e sem bacteremia

\begin{tabular}{|c|c|c|c|}
\hline & $\begin{array}{c}\text { Bacteremia } \\
\text { Média- } \\
\text { Desvio Padrão }\end{array}$ & $\begin{array}{c}\frac{\text { Sem bacteremia }}{\text { Média- }} \\
\text { Desvio Padrão } \\
\end{array}$ & $\mathrm{p}$ \\
\hline Hematócrito(\%) & $40,5 \pm 3,6$ & $39,5 \pm 4,1$ & $0,78 *$ \\
\hline Hemoglobina(g/dL) & $13,03 \pm 1,4$ & $|3| \pm 1,6$, & $0,43 * *$ \\
\hline Leucócitos $\left(\mathrm{mm}^{3}\right)$ & $12.625 \pm 4.629$ & $10.976 \pm 3.685$ & $0,37 *$ \\
\hline Proteína CReativa(mg/L) & $110,0 \pm 96,2$ & $153,4 \pm 81,9$ & 0,30 *** \\
\hline Bilirrubinatotal(mg/dL) & $1,31 \pm 1,2$ & $1,14 \pm 0,9$ & 0,96 *** \\
\hline Bilirrubinadireta(mg/dL) & $1,20 \pm 1,7$ & $0,46 \pm 0,6$ & 0,35 *** \\
\hline Bilirrubina indireta(mg/dL) & $0,93 \pm 0,6$ & $0,70 \pm 0,4$ & 0,30 *** \\
\hline AST (u/L) & $29,12 \pm 14,6$ & $29,42 \pm 41,9$ & $0,38 *$ \\
\hline $\mathrm{ALT}(\mathrm{u} / \mathrm{L})$ & $27,5 \pm 14,3$ & $35,34 \pm 47,1$ & $0,98 *$ \\
\hline Fosfatase alcalina(u/L) & $|5|, 8 \pm \mid 56,2$ & $|45,2 \pm 1| 6,5$ & 0,73 米 \\
\hline Glicemia(mg\%) & $|5|, 5 \pm 132$ & $122,19 \pm 64$ & 0,76 *** \\
\hline Amilase $(u / L)$ & $51,63 \pm 22,0$ & $46,50 \pm 62,6$ & 0,97 米 \\
\hline Gama-glutamil-transferase (u/L) & $56,7 \pm 77,0$ & $78,2 \pm 88,7$ & 0,10 *** \\
\hline Creatinina(mg\%) & $1,57 \pm 0,7$ & $0,95 \pm 0,4$ & $0,028 *$ \\
\hline
\end{tabular}


Tabela 5 - Distribuição da freqüencia de pacientes com complicações nos grupos com e sem bacteremia

\begin{tabular}{|c|c|c|c|}
\hline & Bacteremia (8) & Sem bacteremia (43) & $p$ \\
\hline Complicações infecciosas & 5 & 10 & 0,039 \\
\hline Complicações não infecciosas & 4 & 7 & 0,055 \\
\hline
\end{tabular}

Teste exato de Fisher (p)

\section{Gráfico I - Resultado das hemoculturas - sensibilidade antibiótico (\%)}

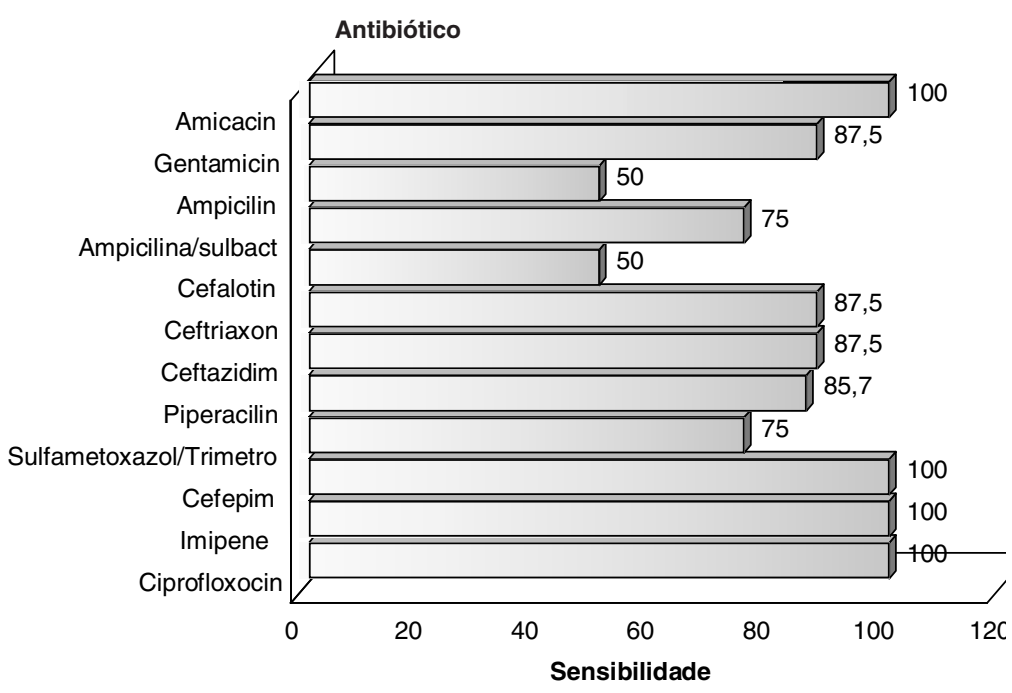

As espécies bacterianas identificadas nas hemoculturas foram exclusivamente Gramnegativas, Identificou-se em quatro pacientes como tipo bacteriano Escherichia coli, e nos outros quatro, Klebsiella sp.

Foram excluídas do grupo bacteremia duas hemoculturas em dois pacientes diferentes. Houve o crescimento de bactérias tipicamente da flora cutânea (Streptococcus epidermidise Staphylococcus coagulase negativo) em uma única amostra. Na cultura da bile de ambos os pacientes, não houve crescimento bacteriano.

A sensibilidade e resistência bacteriana dos resultados das hemoculturas, nos oito pacientes com bacteremia, tanto para E. coli quanto para Klebsiella, estão apresentados resumidamente no Gráfico I.

\section{Discussão}

Vários estudos correlacionam SIRS e sepse com a presença de bacteremia 23,24,25. Sabe-se que a presença da bacteremia pode não ser essencial para a manutenção e evolução do quadro de sepse ${ }^{26}$. A presença e a repercussão clínica da bacteremia nas mais variadas doenças ainda não estão definitivamente determinadas. Vários fatores são determinantes de sua importância clínica, como local da infecção, idade dos pacientes, gravidade da doença primária, tipo de patógeno envolvido, imunidade do paciente $27,28,29$. $O$ espectro de gravidade diante de sua presença pode variar, desde um evento sem repercussões clínicas até altas taxas de complicações e mortalidade ${ }^{30}$.

A prevalência da bacteremia nessa pesquisa foi de 15,68\% (8 em 51 pacientes). Estudos anteriores mostraram uma prevalência que variou de $7,65 \%$ a $12,5 \%$ em pacientes portadores de colecistite aguda litiásica submetidos a tratamento cirúrgico de emergência ${ }^{7,31}$.

Algumas características do nosso estudo relacionadas aos critérios de diagnóstico, seleção da amostra e método utilizado para a realização de hemoculturas devem ter sido as responsáveis pelas diferenças encontradas em relação aos estudos prévios.

Os critérios de diagnóstico clínico e os achados histopatológicos muitas vezes não são correspondentes ${ }^{32}$, não evidenciando os achados de colecistite aguda; além disso, os achados ultra-sonográficos para diagnóstico de colecistite aguda litiásica apresentam baixa especificidade ${ }^{1,33}$. No nosso estudo, o exame padrão definitivo foi o histopatológico da peça operatória.

Em relação ao método para detecção de bacteremia, foi utilizado o sistema Bactec 9240ß) (Becton end Dickinson Diagnostic Instruments Systems), sistema automatizado, sensível no diagnóstico quando comparado a métodos manuais e outros sistemas automatizados $^{17,18}$, que apresenta menor tempo de detecção do crescimento de bactérias nas hemoculturas, possibilita menor manipulação após a incubação, monitoração contínua e capacidade de acomodar um grande número de frascos ao mesmo tempo ${ }^{18}$.

Os frascos do sistema Bactec utilizam resina para adsorção de antibióticos, isto possibilita melhorar os índices de recuperação de bactérias, mesmo em pacientes que não estejam em uso de antibióticos. A agitação constante do meio na presença de resinas promove lise das hemáceas e leucócitos e cria superfícies, nas quais as bactérias se aderem, melhorando as condições para a sua reprodução. Outro fator importante é o volume de sangue utilizado em cada frasco nas coletas dos mesmos. Em cada frasco são coletados $10 \mathrm{ml}$ de sangue, o que aumenta a sensibilidade do método ${ }^{15,16}$; sabe-se que em adultos, cada $\mathrm{ml}$ de sangue cultivado aumenta em cerca de $3 \%$ a positividade das hemoculturas ${ }^{16}$.

Em relação à amostra de pacientes em nosso estudo, observou-se uma freqüência de $35,7 \%$ de idosos acima de 60 anos e uma média de idade no grupo com a presença de bacteremia de 68,5 anos. Os pacientes idosos de maneira geral possuem uma maior freqüência de bacteremia como mostrado em estudos prévios $^{34,35}$

Chetlin \& Elliot ( $97 \mid)^{36}$ detectaram em 80 casos de bactibilia em portadores de colecistite aguda litiásica, associados ou não a coledocolitíase e icterícia obstrutiva, 12,5\% de bacteremia. A maioria destes pacientes apresentava mais de 70 anos (72\%). Foi utilizado critério histopatológico de colecistite aguda ou gangrena. Não existem referências ao tipo de técnica, número e momento exato da coleta das hemoculturas.

Estudo realizado por Keighley et al. (1976) ${ }^{7}$ observou no segmento dos pacientes submetidos à cirurgia de emergência uma freqüência de bacteremia de $12 \%$ nos colecistecto- 
mizados. Não existem no referido estudo dados quanto aos métodos utilizados na realização das culturas nem os critérios definitivos para o diagnóstico de colecistite aguda.

Estudo realizado por Kuo et al. (1995)31 avaliou a presença de bacteremia em portadores de colecistite aguda e observou uma prevalência de 7,65\%, porém, utilizou somente critérios clínicos e ultra-sonográficos para estabelecer o diagnóstico de colecistite aguda; isso implica no risco de inclusão de casos com diagnóstico incorreto. Utilizou método manual e com menor volume de sangue $(5 \mathrm{ml})$ para a realização das hemoculturas. Além disso, a média de idade da amostra dos pacientes com bacteremia foi inferior à do nosso estudo.

Kuo et al. $(1995)^{31}$, em estudo prospectivo, observaram em pacientes portadores de colecistite aguda bacterêmica que os mais idosos apresentaram pior prognóstico e uma maior incidência de mortalidade.

A bacteremia tem sido associada a vários fatores além da idade, dentre eles as doenças prévias e uma baixa reserva fisiológica ${ }^{25,35}$. Freqüentemente presentes na população ido$\mathrm{sa}^{37}$, esses fatores podem contribuir para uma maior prevalência de bacteremia nos idosos.

Em pacientes portadores de colecistite aguda litiásica, a idade avançada está associada a uma maior taxa de complicações infeccio$\operatorname{sas}^{3,4,38,39}$ e maior prevalência de bactibilia ${ }^{4,7,38}$. Houve, no nosso estudo, uma alta prevalência de pacientes idosos no grupo bacteremia (87,5\%). Assim, acreditamos que os idosos portadores de colecistite aguda litiásica por apresentarem geralmente doença em fase mais avançada, maior freqüência de doenças associadas e uma pior resposta imune tanto celular como humora ${ }^{37}$ estão mais sujeitos a apresentar bacteremia.

A análise das médias da freqüência cardíaca e da freqüência respiratória e os sinais de SIRS, entre os grupos com e sem bacteremia, mostraram significante associação com bacteremia, com respectivamente $p=0,026, p=$ 0,028 e $p=0,016$.

O aumento da freqüência cardíaca como medida isolada é um achado inespecífico podendo ocorrer a uma série de estímulos não infecciosos. Contudo, o aumento da freqüência cardíaca pode ocorrer antes de alteração no número de leucócitos, temperatura e pressão arteria| ${ }^{40}$. Portanto, taquicardia é sinal precoce e sensível de infecção, mas apresenta baixa especificidade.

Peduzzi et al. (19924) estabelecem como um dos fatores associados à bacteremia 0 aumento precoce na freqüência respiratória em pacientes com diagnóstico de sepse, que pode aparecer mesmo em ausência de febre e leucocitose.

Estudos prévios de Bossink et al. (1999)25 observaram que pacientes com sinais de sepse estabelecidos por Bone et al. (1992) ${ }^{10}$ apresentam alta sensibilidade para o diagnóstico de infecção e bacteremia. Os mesmos autores sugerem que novos parâmetros sejam utilizados para aumentar a especificidade dos critérios atualmente utilizados no diagnóstico de sepse, e que taquicardia e taquipnéia sejam trocadas por outros achados clínicos e laboratoriais com o propósito de tornar os critérios mais específicos.

Estudo realizado por Jones \& Lowes $(1996)^{24}$, em condições clínicas diversas, encontrou uma sensibilidade de SIRS para detectar bacteremia de 95\%, entretanto, a maioria dos pacientes com SIRS não apresentava bacteremia, e o valor preditivo positivo para SIRS, como preditor de bacteremia, era somente de $7 \%$.

A bacteremia secundária a um processo infeccioso focal geralmente indica uma maior gravidade ${ }^{35,42}$ e com freqüência um maior estímulo de resposta inflamatória sistêmica ${ }^{43}$, assim, o estado bacterêmico pode estar associado a uma maior prevalência ou acentuação dos sinais de resposta inflamatória sistêmica.

Os nossos resultados em relação à SIRS no diagnóstico de bacteremia mostram-se semelhantes a estudos anteriores relatados que utilizaram os mesmos critérios em diversas doenças. A utilização dos critérios de SIRS, em portadores de colecistite aguda litiásica, mostrou-se mais útil para afastar a bacteremia do que para determinar a sua presença.

O estudo mostrou uma associação significante de bacteremia com elevação dos níveis de creatinina $(p=0,028)$. A elevação dos níveis de creatinina está associada à maior prevalência de bacteremia em estudos com pacientes com infecções de etiologia e foco primário variados ${ }^{42,44}$. Além disso, o aumento dos níveis de creatinina em pacientes que apresentavam níveis previamente normais está associado a uma maior mortalidade em pacientes com sepse $e^{45}$.
Estes achados podem ter relação com o fato de que pacientes bacterêmicos podem apresentar quadros infecciosos mais graves e uma resposta inflamatória sistêmica mais acentuada, provocando uma pior perfusão tecidual, com conseqüente piora da perfusão renal e elevação dos níveis de creatinina ${ }^{46}$.

Outro fator que pode ter influenciado nos resultados é a alta prevalência de idosos portadores de bacteremia neste estudo, que freqüentemente apresentam baixa reserva fisiológica $^{37}$, e podem desenvolver com mais freqüência elevação dos níveis de creatinina em vigência de infecção do trato biliar.

Ocorreu um número significantemente maior de complicações pós-operatórias de maneira global e complicações infecciosas no grupo com bacteremia, determinando, assim, um maior período de internação hospitalar. Um paciente faleceu na casuística; pertencente ao grupo com bacteremia, apresentou complicações cardíacas que evoluíram com insuficiência de múltiplos órgãos. Apesar disso, não se pode caracterizar a causa do óbito como diretamente relacionada à presença de bacteremia, pois o paciente já apresentava doença cardíaca prévia de gravidade considerável.

Estudos prévios em pacientes em estado crítico estabeleceram relação da bacteremia e sepse com maior tempo de internação, elevação dos custos e maior número de complicações ${ }^{11,47,48}$.

Kuo et al. (1995)31, em estudo prospectivo em pacientes portadores de colecistite aguda litiásica com e sem bacteremia, evidenciaram um aumento de complicações sistêmicas e mortalidade em portadores de bacteremia. Bedirli et al. (200 I $)^{49}$ determinaram em pacientes portadores de colecistite aguda, submetidos a colecistectomia, que os fatores de risco para o desenvolvimento de complicações são a idade avançada, presença de doenças concomitantes, disfunção circulatória, febre e leucocitose.

Em nosso estudo, não podemos afirmar que a bacteremia foi o fator determinante do maior número de complicações. Como qualquer realidade clínica é complexa, a possibilidade de fatores de confusão deve ser sempre considerada; na maioria das vezes, múltiplos fatores são responsáveis pelos resultados obti$\operatorname{dos}^{50}$. Assim, apesar do pequeno número da nossa casuística no grupo bacteremia (oito pacientes), os dados sugerem uma 
Battello Al et al.

tendência para caracterizar um grupo de pacientes com maior gravidade e maior risco de complicações no pós-operatório e, como referido por Raymond $(200 \mathrm{l})^{30}$, que avaliou a bacteremia em pacientes cirúrgicos e infectados, esta é um marcador de gravidade da doença. Portanto, nos portadores de colecistite aguda litiásica, a bacteremia pode estar associada a uma maior gravidade e à maior tendência do surgimento de complicações no pós-operatório promovendo, maior tempo de internação.

As bactérias identificadas nas hemoculturas foram Gram-negativas: Escherichia coli e Klebsiella sp., semelhantes aos estudos prévios de Siegman-|gra (1988)51 e Kuo (1995)31, que encontraram predominantemente bactérias Gram-negativas do tipo enterobactérias. Esses resultados são esperados, pois são as bactérias mais freqüentemente encontradas no conteúdo biliar em colecistite aguda litiásica ${ }^{3,5}$. Em relação ao teste de sensibilidade das bactérias encontradas nas hemoculturas, encontramos uma alta sensibilidade à maioria dos antibióticos testados (aminoglicosídeo, cefalosporinas de terceira e quarta geração, piperacilina, imipenem e ciprofloxacina). A amostra de oito pacientes no grupo bacteremia não permite conclusões definitivas, mas podemos apontar para uma tendência de caracterização dos microrganismos envolvidos como sendo bactérias Gram-negativas com alta sensibilidade a antibióticos, como aminoglicosídeos, cefalosporinas de terceira e quarta geração.

Acreditamos que a presença de bacteremia está associada a uma maior gravidade nos portadores de colecistite aguda litiásica. A detecção de fatores associados à presença de bacteremia poderia auxiliar a conduta terapêutica desses pacientes, permitindo aplicação de cuidados clínicos intensivos e a antibioticoterapia precoce e específica, possivelmente reduzindo as complicações e mortalidade. Por outro lado, nos portadores de colecistite aguda litiásica sem sinais de SIRS, evidenciando baixa probabilidade de bacteremia, poderia ser evitado o uso desnecessário e prolongado de antibióticos.

\section{Conclusões}

Em nosso estudo, a prevalência de bacteremia no pré-operatório de portadores de colecistite aguda litiásica foi considerável (I 5,68\%). A presença de Síndrome da Resposta Inflamatória Sistêmica e o aumento da freqüência cardíaca, da frequêencia respiratória e da creatinina associam-se, significantemente, à presença de bacteremia.

O número de pacientes com complicações e o tempo de internação são significantemente maiores nos portadores de bacteremia, ocorrendo neste mesmo grupo o único óbito. A presença de bacteremia está relacionada a um pior prognóstico e caracterizou um grupo de maior gravidade.

Conflito de interesse: não há.

\section{SUMMARY}

\section{BACTEREMIA - PREVALENCE AND ASSOCIATED FACTORS IN PATIENTS WITH ACUTE CALCULOUS CHOLECYSTITIS}

BACKGROUND. Bacteremia seems to increase the morbidity and mortality in clinical and surgical conditions, however the importance and factors associated with it during acute calculous cholecystitis are not well established. The purpose of this study was to determine pre-operative prevalence, associated factors and prognosis of bacteremia in patients with acute calculous cholecystitis submitted to an emergency cholecystectomy.

METHODS. A prospective study of 5 / patients with histological diagnosis of acute calculous cholecystitis was designed. The Bactec System was used to detect bacteremia. Mean results of clinical and laboratory analyses were related to the presence of bacteremia.

RESULTS. The pre-operative prevalence of bacteremia was of $15.68 \%$. Age $(P=0.024)$, heart rate $(P=0.026)$, respiratory rate $(P=0.028)$, serum creatinine $(P=0.028)$ and presence of systemic inflammatory response syndrome $(P=0.016)$ were positively associated with bacteremia. Bacteremic patients had statistically higher overall $(P=0.045)$ and infectious $(P=0.039)$ complication rates and longer hospitalization $(P<0.005)$ including one death.

Conclusions. Patients with acute calculous cholecystitis have a considerable prevalence of bacteremia in the pre-operative period. It is associated with old age, heart rate, respiratory rate, serum creatinine and systemic inflammatory response syndrome. In patients with acute calculous cholecystitis, presence of bacteremia may be associated to severity of the condition and poor outcome related to pos-operative complications. [Rev Assoc Med Bras 2004; 50(4): 373-9]

KeY wORDS: Bacteremia. Prevalence. Acute cholecystitis. SIRS and complications.

\section{REFERÊNCIAS}

I. Sharp KW. Acute cholecystitis. Surg Clin North Am 1988:68(2):269-79.

2. Järvinen HJ. Biliary bacteremia at various stages of acute cholecistitis. Acta Chir Scand 1980; I 46(6):427-30.

3. Thompson JE, Bennion RS, Doty JE, Muller $E L$, Pitt HA. Predictive factors for bactibilia in acute cholecytitis. Arch Surg 1990; 125(2):261-4.

4. Claesson B, Holmlund D, Matzsch T. Biliary microflora in acute cholecystitis and the clinical implications. Acta Chir Scand 1984; 150(3):229-37

5. Farinon AM, Grande M, Torquati A, D'antini $P$. Multivariate analysis for predicting the presence of bacteria in bile in patients with acute cholecystitis. Eur J Surg 1993; 159(10):531-4.

6. Csendes A, Burdiles P, Maluenda F, Diaz JC, Csendes $P$, Mitru N. Simultaneous bacteriologic assessment of bile from gallbladder and common bile duct in control subjects and patients with gallstones and common duct stones. Arch Surg 1996; I3 I(4):389-94.

7. Keighley MR, Flinn R, Alexander-Williams J. Multivariate analysis of clinical and operative findings associated with biliary sepsis. Br J Surg 1976; 63(7):528-31.

8. Bone RC. Toward an epidemiology and natural history of SIRS (systemic inflammatory response syndrome). JAMA 1992; 268(24): 3452-5.

9. Aronson MD, Bor DH. Blood cultures. Ann Intern Med 1987; 106(2):246-53.

10. Bone RC, Balk RA, Cerra FB, Dellinger RP, Fein AM, Knaus WA, et al. Definitions for sepsis and organ failure and guidelines for the use of innovative therapies in sepsis. Chest 1992; I0 I (6): I 644-55.

II. Pittet D, Tarara D, Wenzel RP. Nosocomial bloodstream Infection in critically ill patients. Excess length of stay, extra costs, and attributable mortality. JAMA 1994; 27 I (20): | 598-60 I

12. Bates DW, Pruess KE, Lee TH. How bad are bacteremia and sepsis? Outcomes in a cohort with suspected bacteremia. Arch Intern Med 1995; 155(6):593-8.

13. Weinstein MP, Towns ML, Quartey SM, Mirrett S, Reimer LG, Parmigiani G, et al. The clinical significance of positive blood cultures in the 1990s: a prospective comprehensive evaluation of the microbiology epidemiology, and outcome of bacteremia and fungemia in adults. Clin Infect Dis 1997; 24(4):584-602. 
14. Koontz FP, Flint KK, Reynolds JK, Allen SD. Multicenter comparison of the high volume ( 10 $\mathrm{ml)}$ NR BACTEC PLUS system and the standard $(5 \mathrm{ml})$ NR BACTEC system. Diagn Microbiol Infect Dis 199 |; | 4(2): I I -8.

15. Porter RC, Lo P, Low DE, Simor AE, McGeer $A$, Scriver $S$, et al. Utilization review of the use the BACTEC PLUS high - volume blood cultures bottles. J Clin Microbiol 1993; 3 I ( I0):2794-5.

16. Mermel LA, Maki DG. Detection of bacteremia in adults: consequences of culturing an inadequate volume of blood. Ann Intern Med 1993; I I9(4):270-2

17. Nolte FS, Williams JM, Jerris RC, Morello JA, Leitch CD, Matushek S, et al. Multicenter clinical evaluation of a continuous monitoring blood culture system using fluorescent-sensor technology (BACTEC 9240). J Clin Microbiol 1993; 31 (3):552-7

18. Koneman EW. Diagnóstico microbiológico. $5^{\mathrm{a}}$ ed. Rio de Janeiro: Medsi; 2001.

19. Sariego J, Matsumoto T, Kerstein M. Significance of wall thickness in symptomatic gallbladder disease. Arch Surg 1992; 127(10): 1216-8

20. Scott HS. Gallblader and extra hepatic biliary tree. In: Stermberg SS, editor. Diagnostic surgical pathology. $2^{\text {a }}$ ed. New York: Raven Press; 1994. p. I582-6.

21. MacGregor RR, Beaty HN. Evaluation of positive blood cultures. Guidelines for early differentiation of contamined from valid positive cultures. Arch Intern Med 1972; |30(I):84-7.

22. Weinstein MP, Murphy JR, Reller LB, Lichtenstein KA. The clinical significance of positive blood cultures: a comprehensive analysis of 500 episodes of bacteremia and fungemia in adults. II. Clinical observations, with special reference to factors influencing prognosis. Rev Infect Dis 1983; 5(1):54-70.

23. Aube $H$, Milan $C$, Blettery B. Risk factors for septic shock in the early management of bacteremia. Am J Med 1992; 93(3):283-8.

24. Jones GR, Lowes JA. The systemic inflammatory response syndrome as a predictor of bacteremia and outcome from sepsis. QJM 1996; 89(7):5 I5-22

25. Bossink AW, Groeneveld AB, Hack CE, Thijs LG. The clinical host response to microbial infection in medical patients with fever Chest 1999; I 16(2):380-90.

26. Rangel-Frausto MS, Pittet D, Costigan M, Hwang T, Davis CS, Wenzel RP. The natural history of the systemic inflammatory response syndrome (SIRS). A prospective study. JAMA 1995; 273(2): 1 17-23.

27. Brun-Buisson C, Doyon F, Carlet]. Bacteremia and severe sepsis in adults: a multicenter prospective surgery in ICUs and wards of 24 hospitals. French Bacteremia Sepsis Study Group. Am J Respir Crit Care Med 1996; 154(3 Pt l):6 |7-24.

28. Torres-Tortosa M, Canueto J, Bascunana A, Vergara A, Sanchez-Porto A, MorenoMaqueda I, et al. Prognostic evaluation of bacteremia and fungemia in patients with acquired immunodeficiency syndrome. Eur. J Clin Microbiol Infect Dis 2002; 2 I (4):262-8.

29. Vales EC, Abraira V, Sánchez JC, García MP, Feijoo AR, Alvarez M, et al. A predictive model for mortality of bloodstream infections. Bedside analysis with the Weibull function. J Clin Epidemiol 2002; 55(6):563-72.

30. Raymond DP, Pelletier SJ, Crabtree TD, Gleason TG, Pruett TL, Sawyer RG. Impact of bloodstream Infection on outcomes among infected surgical inpatients. Ann Surg 200I; 233(4):549-53.

31. Kuo CH, Changchien CS, Chen JJ, Tai DI, Chiou SS, Lee CM. Septic acute cholecystitis. Scand J Gastroenterol 1995; 30(3):272-5.

32. Sianesi M, Ghirarduzzi A, Percudani M, Dell'Anna B. Cholecistectomy for acute cholecystitis: timing of operation, bacteriologic aspects and postoperative course. Am J Surg 1984; | 48(5):609-12.

33. Carroll BA. Preferred imaging techniques for the diagnosis of cholecystitis and cholelithiasis. Ann Surg 1989; 2 10(I): I- 12 .

34. Kreger BE, Craven DE, Carling PC, McCabe WR. Gram-negative bacteremia III. Reassessment of etiology, epidemiology and ecology in 612 patients. Am J Med 1980; 68(3):332-43.

35. Bates DW, Cook EF, Goldman L, Lee TH. Predicting bacteremia in hospitalized patients. A prospectively validated model. Ann Intern Med 1990; I I3(7):495-500.

36. Cheltin SH, Elliot DW. Biliary bacteremia. Arch Surg 1971;102(4):303-7.

37. Meyers BR, Sherman E, Mendelson MH, Velasquez G, Srulevitch-Chin E, Hubbard M, et al. Bloodstream infections in the elderly. Am J Med 1989; 86(4):379-84.

38. Pitt HA, Postier RG, Cameron JL. Biliary bacteria: significance and alterations after antibiotic therapy. Arch Surg 1982; I 17(4):445-9.
39. Tokunaga $Y$, Nakayama N, Ishikawa $Y$, Nishitai $R$, Irie A, Kaganoi J, et al. Surgical risks of acute cholecystitis in elderly. Hepatogastroenterology 1997; 44(1 5):67 I-6.

40. Chandler CF, Waxman K. Monitoramento. In: Grenvik A. Manual de terapia intensiva. São Paulo: Roca Editora; 1998. p.64-98.

41. Peduzzi P, Shatney C, Sheagren J, Sprung C. Predictors of bacteremia and gram-negative bacteremia in patients with sepsis. Arch Intern Med 1992; I 52(3):529-35.

42. Leibovici L, Greenshtain S, Cohen O, Mor F, Wysenbeek AJ. Bacteremia in febrile patients. A clinical model for diagnosis. Arch Interm Med |99|; | 5 |(9): | 80 |-6

43. Bone RC, Grodzin CJ, Balk RA. Sepsis: a new hypothesis for pathogenesis of the disease process. Chest 1997; I I2(I):235-43.

44. Gleckman R, Hibert D. Afebrile bacteremia. A phenomenon in geriatric patients. JAMA 1982; 248(I2):|478-8|.

45. Pittet D, Thiévent B, Wenzel RP, Li N, Auckenthaler R, Suter PM. Bedside prediction of mortalily from bacteremic sepsis. A dynamic analysis of ICU patients. Am J Respir Crit Care Med 1996; I 53(2):684-693.

46. Bossink AW, Groeneveld AB, Koffeman GI, Becker A. Prediction of shock in febrile medical patients with a clinical infection. Crit Care Med 200 I; 29(I):25-3I.

47. Smith RL, Meixler SM, Simberkoff MS. Excess mortality in critically ill patients with nosocomial bloodstream infections. Chest 1991; 100(I):164-7.

48. Leibovici L, Samra Z, Konisberger H, Drucker $M$, Ashkenazi S, Pitlik SD. Long-term surgical following bacteremia or fungemia. JAMA 1995; 274(10):807-12

49. Berdili A, Sakrak O, Sözuer EM, Kerek M, Güler I. Factors effecting the complications in the natural history of acute cholecystitis. Hepatogastroenterology 200 I; 48(4I): 1275-8.

50. Soares JF, Siqueira AL. Introdução à estatística médica. $2^{a}$ ed. Belo Horizonte: Coopmed; 2002

5I. Siegman-Igra Y, Schwatz D, Konforti N, Perluk C, Rozin RR. Septicemia from biliary tract infection. Arch Surg 1988; 123(3):366-8.

Artigo recebido: 29/10/03

Aceito para publicação: 18/05/04 